\title{
Power allocation for maximizing the MAC capacity via majorization
}

\author{
Jisheng Dai ${ }^{1,2,3}$ and Chunqi Chang ${ }^{4^{*}}$
}

\begin{abstract}
Optimal power allocation for maximizing the sum capacity of multiple access channel (MAC) with quality-of-service (QoS) constraints is investigated in this paper. Majorization theory is the underlying mathematical theory on which our method hinges. It is shown that the optimal structure of the solution can be easily obtained via majorization theory. Furthermore, based on our new approach, an efficient searching method for power allocation is developed by restricting the attention to a new searching variable. Our new method requires less than half of the computational cost of the existing method in the worst case and is even much faster in general. Simulation results demonstrate the effectiveness of our method.
\end{abstract}

Keywords: Multiple access channel (MAC); Power allocation; Capacity; Quality-of-service (QoS)

\section{Introduction}

In order to interface to broadband networks of the future, wireless system will be required to support real-time and non-real-time multi-rate services [1,2]. For the real-time services having little tolerance for delay, such as voice and low-rate video, rate adaptation is not desirable, while for the non-real-time services, such as data communication (paging, electronic mail, etc.), rate adaptation is allowed since they are tolerant to latencies though sensitive to transmission errors. It is well known that the radio spectrum can be utilized more efficiently if the delay-tolerant traffic users are managed with an optimal resource allocation policy [3-6].

In this paper, we are more interested in the optimal power allocation policy that maximizes sum capacity of non-real-time users in multiple access channel (MAC). It has captured considerable attention during the past years. Maximizing the reverse link (uplink) sum capacity of non-real-time users has been studied in [2]. However, the maximum sum capacity is usually achieved via unfair resource allocation that ignores the throughput from users with poor channel conditions. To avoid this situation, additional quality-of-service (QoS) requirements are introduced to practical systems. A method to obtain

*Correspondence: cqchang@suda.edu.cn

${ }^{4}$ School of Electronic and Information Engineering, Soochow University, Suzhou 215006, China

Full list of author information is available at the end of the article the maximal reverse link sum capacity, under some QoS constraints, is proposed in $[3,4]$. It is shown that the optimal solution can be obtained by a numerical search over a finite number of one-dimensional search spaces. In order to reduce the computational cost, an improved method is developed to eliminate the numerical search [5]. With the introduction of some specific hyperplanes, it is shown that the candidate solutions can be determined in a closed form so that the search space is reduced into a finite set of points. However, the main theorem in [5] is explained by graphical visualization only, instead of strict mathematical proof. Consequently, some important relationships are not revealed properly.

Inspired by the treatment proposed in [6], we try to refine the results in [3-5] via majorization theory, where the proof procedure can be adapted to a great number of design criteria, as long as their objective functions are Schur-concave or Schur-convex [7-9]. It turns out from our new derivation that the size of the search space in the original method can be further reduced if we change the search variable and rearrange the constraints. Compared to the existing method, less than half of the computational cost is required in the worst case.

\section{Preliminaries}

In this section, we introduce the basic notations and a lemma of majorization, which will be used in Section 4 .

\section{Springer}


For a detailed description of majorization, the readers are referred to [8] and [9].

Definition 1. For any vector $\mathbf{x}=\left[x_{1}, x_{2}, \cdots, x_{n}\right]^{T} \in \mathbb{R}^{n}$, let $x_{[1]} \geq x_{[2]} \geq \cdots \geq x_{[n]}$ denote its components in a non-increasing order, and let

$$
\mathbf{x}_{\downarrow}=\left[x_{[1]}, x_{[2]}, \cdots, x_{[n]}\right]^{T}
$$

denote the decreasing rearrangement of $\mathbf{x}$.

Definition 2. For any two vectors $\mathbf{x}, \mathbf{y} \in \mathbb{R}^{n}$, we say $\mathbf{x}$ is majorized by $\mathbf{y}$ if

$$
\begin{aligned}
& \sum_{i=1}^{k} x_{[i]} \leq \sum_{i=1}^{k} y_{[i]}, \quad 1 \leq k \leq n-1, \\
& \sum_{i=1}^{n} x_{[i]}=\sum_{i=1}^{n} y_{[i]} .
\end{aligned}
$$

and we represent it by $\mathbf{x} \prec \mathbf{y}$.

Definition 3. A real-valued function $\phi$ defined on a set $\mathcal{A} \subseteq \mathbb{R}^{n}$ is said to be Schur-convex on $\mathcal{A}$ if

$$
\mathbf{x} \prec \mathbf{y} \text { on } \mathcal{A} \Rightarrow \phi(\mathbf{x}) \leq \phi(\mathbf{y}) .
$$

Similarly, $\phi$ is said to be Schur-concave on $\mathcal{A}$ if

$$
\mathbf{x} \prec \mathbf{y} \text { on } \mathcal{A} \Rightarrow \phi(\mathbf{x}) \geq \phi(\mathbf{y}) \text {. }
$$

Lemma 1. $f(\mathbf{x})=\prod_{i} x_{i}$ is Schur-concave on the set of $\left\{\mathbf{x} \mid \forall i, x_{i} \geq 0\right\}$.

Proof. Assume $\mathbf{x}_{1}, \mathbf{x}_{2} \in \mathbb{R}^{n}$ with $\mathbf{x}_{1} \prec \mathbf{x}_{2}$. According to B.1. Lemma in [8], $\mathbf{x}_{1}$ can be derived from $\mathbf{x}_{2}$ by successive applications of a finite number of $T$ transforms, e.g.,

$$
\mathbf{x}_{1}=\mathbf{T}_{1} \mathbf{T}_{2} \cdots \mathbf{T}_{m} \mathbf{x}_{2}
$$

where $m$ is a positive integer and $T_{i} \mathrm{~s}, i=1,2, \ldots, m$, are the $T$-transform matrices. Each $T$-transform matrix has the form of $\lambda \mathbf{I}+(1-\lambda) \mathbf{Q}$, where $0 \leq \lambda \leq 1$ and $\mathbf{Q}$ is a permutation matrix that just interchanges two coordinates. Obviously, if $f(\mathbf{T x}) \geq f(\mathbf{x})$ holds for any $T$-transform matrix $\mathbf{T}$, we will achieve the desired result $f\left(\mathbf{x}_{1}\right) \geq f\left(\mathbf{x}_{2}\right)$. The reason is given as follows:

$$
\begin{aligned}
f\left(\mathbf{x}_{2}\right) & \leq f\left(\mathbf{T}_{m} \mathbf{x}_{2}\right) \leq f\left(\mathbf{T}_{m-1} \mathbf{T}_{m} \mathbf{x}_{2}\right) \leq \cdots \\
& \leq f\left(\mathbf{T}_{1} \mathbf{T}_{2} \cdots \mathbf{T}_{m} \mathbf{x}_{2}\right)=f\left(\mathbf{x}_{1}\right) .
\end{aligned}
$$

What remains is to prove $f(\mathbf{T} \mathbf{x}) \geq f(\mathbf{x})$. Without loss of generality, assume that the permutation matrix interchanges the $j$ th and $k$ th coordinates. Then, we have

$$
[\mathbf{T x}]_{i}= \begin{cases}x_{i} & i \neq j, k \\ \lambda x_{j}+(1-\lambda) x_{k}, & i=j \\ \lambda x_{k}+(1-\lambda) x_{j}, & i=k\end{cases}
$$

where $[\cdot]_{i}$ stands for the $i$ th element of a vector. With (3), we obtain:

$$
\begin{aligned}
f(\mathbf{T x}) & =\left(\prod_{i \neq j, k} x_{i}\right) \cdot\left(\lambda x_{j}+(1-\lambda) x_{k}\right)\left(\lambda x_{k}+(1-\lambda) x_{j}\right) \\
& =\left(\prod_{i \neq j, k} x_{i}\right)\left(\lambda(1-\lambda)\left(x_{j}-x_{j}\right)^{2}+x_{j} x_{k}\right) \\
& =\left(\prod_{i \neq j, k} x_{i}\right) \lambda(1-\lambda)\left(x_{j}-x_{j}\right)^{2}+\left(\prod_{i} x_{i}\right) \\
& \geq f(\mathbf{x}) .
\end{aligned}
$$

\section{Problem formulation}

Consider a MAC wireless network supporting $M$ data mobile stations (MS), whose link gains are $g_{1}, g_{2}, \cdots, g_{M}$. Without loss of generality, assume $g_{1} \geq g_{2} \geq \cdots \geq g_{M} \geq$ 0 . Defining $p_{i}$ as the $i$ th MS's transmit power, the signalto-interference-plus-noise ratio (SINR) for the $i$ th MS at the base station is given by

$$
\operatorname{SINR}_{i}=\frac{p_{i} g_{i}}{I+\sum_{j=1, j \neq i}^{M} p_{j} g_{j}}, i=1,2, \cdots, M
$$

where $I$ is the power of the background noise and interferences from other traffic-type users. Note that the BS is assumed to independently decode different users, i.e., it is not using a successive cancelation scheme at the receiver. From Shannon formula, the sum capacity of data users in MAC becomes:

$$
C=\log _{2} \frac{\left(I+\sum_{j=1}^{M} p_{j} g_{j}\right)^{M}}{\prod_{i=1}^{M}\left(I+\sum_{j=1, j \neq i}^{M} p_{j} g_{j}\right)}
$$

Our goal is to derive an optimal power allocation that maximizes the sum capacity of the data users. There are generally three constraints that must be satisfied. First, the transmission power at node $i$ must not exceed a certain maximum power $p_{\max }$, which is set by the hardware. Second, the SINR of link $i$ must be above a certain minimum SINR $\gamma$, which is determined by QoS requirements. The third constraint is that the summation of interferences generated from $M$ data users is limited by $\sum_{i=1}^{M} p_{i} g_{i} \leq$ $P_{R}^{\max }$. This constraint is due to the fact that the total 
received power from the data users is an interference to other classes of users, like the voice users in the same cell [3-5]. Note that the way to obtain $P_{R}^{\max }$ is described in [10]. Thus, the classical QoS-constrained optimization problem is formulated as

$$
\max _{\mathbf{p}} \log _{2} \frac{\left(I+\sum_{j=1}^{M} p_{j} g_{j}\right)^{M}}{\prod_{i=1}^{M}\left(I+\sum_{j=1, j \neq i}^{M} p_{j} g_{j}\right)},
$$

subject to : $\forall i, 0 \leq p_{i} \leq p_{\max }$

$$
\begin{aligned}
& \forall i, \frac{p_{i} g_{i}}{I+\sum_{j=1, j \neq i}^{M} p_{j} g_{j}} \geq \gamma \\
& \sum_{i=1}^{M} p_{i} g_{i} \leq P_{R}^{\max }
\end{aligned}
$$

where $\mathbf{p} \triangleq\left[p_{1}, p_{2}, \cdots, p_{M}\right]^{T}$.

\section{Maximizing the sum capacity with QoS constraints}

In this section, we first try to review the optimal structure of the power allocation via majorization theory. Then, we present a fast algorithm by restricting the attention to a new searching variable, and we show that our new method requires less than half of the computational cost of the existing method in the worst case and is even much faster in general.

\subsection{Review of the optimal structure of the power allocation via majorization theory}

Let $x_{i} \triangleq p_{i} g_{i} / I$ and $T \triangleq \sum_{j=1}^{M} x_{j}$, problem (6) can be equivalently rewritten as

$$
\min _{\mathbf{x}, T} \prod_{i=1}^{M} \frac{1+T-x_{i}}{1+T}
$$

subject to : $\forall i, \varphi(1+T) \leq x_{i} \leq l_{i}$

$$
\sum_{i=1}^{M} x_{i}=T, \quad 0 \leq T \leq X_{\max }
$$

where $\mathbf{x} \triangleq\left[x_{1}, x_{2}, \cdots, x_{M}\right]^{T}, l_{i} \triangleq g_{i} p_{\max } / I, \varphi \triangleq \gamma /(\gamma+1)$, $X_{\max } \triangleq P_{R}^{\max } / I$. Note that in deriving (7), the logarithm part is dropped because of its monotonicity. This optimization problem is rather difficult to solve directly, due to its non-convexity. However, if the value of $T$ is fixed, it is possible to solve the optimization problem via majorization theory in a specific hyperplane described by a linear equation $\sum_{j=1}^{M} x_{j}=T$. After that, we are able to find the optimal solution by performing a one-dimensional search on $T$. Defining $y_{i} \triangleq \frac{1+T-x_{i}}{1+T}$, the corresponding problem becomes

$$
\min _{\mathbf{y}} \prod_{i=1}^{M} y_{i}=\min _{\mathbf{y}} f(\mathbf{y})
$$

subject to : $\forall i, \max \left\{\frac{1+T-l_{i}}{1+T}, 0\right\} \leq y_{i} \leq 1-\varphi$

$$
\sum_{i=1}^{M} y_{i}=M-\frac{T}{1+T}, \quad 0 \leq T \leq X_{\max }
$$

where $\mathbf{y} \triangleq\left[y_{1}, y_{2}, \cdots, y_{M}\right]^{T}$ and $f: \mathbf{y} \mapsto \prod_{i=1}^{M} y_{i}$. Since $\sum_{i=1}^{M} y_{i}$ is a constant for fixed $T$, according to Schurconcavity of $f(\mathbf{y})$ (see Lemma 1 ), it is easy to see that

$$
\mathbf{y}^{*} \succ \mathbf{y} \Rightarrow f\left(\mathbf{y}^{*}\right) \leq f(\mathbf{y})
$$

where $\mathbf{y}^{*} \triangleq\left[y_{1}^{*}, y_{2}^{*}, \cdots, y_{M}^{*}\right]^{T}$ and $\sum_{i=1}^{M} y_{i}^{*}=M-\frac{T}{1+T}$. Therefore, solving the optimization problem (8) for fixed $T$ is equivalent to finding the optimal vector $\mathbf{y}^{*}$ such that $\mathbf{y}^{*} \succ \mathbf{y}$ for any feasible $\mathbf{y}$. The corresponding $\mathbf{y}^{*}$ can be explicitly determined as follows.

Theorem 1. For any fixed $T$, the optimal $\mathbf{y}^{*}$ among the constraints of problem (8) must be structured as

$$
\mathbf{y}^{*}=[\underbrace{\tilde{l}_{1}, \cdots, \tilde{l}_{k-1}}_{k-1}, y_{k}, \underbrace{1-\varphi, \cdots, 1-\varphi}_{M-k}]^{T}
$$

where $\tilde{l}_{i} \triangleq \frac{1+T-l_{i}}{1+T}$, the value of $k$ is determined by the following inequality

$$
\sum_{i=k+1}^{M}\left(w-\tilde{l}_{i}\right)<M-\frac{T}{1+T}-\sum_{i=1}^{M} \tilde{l}_{i} \leq \sum_{i=k}^{M}\left(w-\tilde{l}_{i}\right)
$$

and $y_{k}$ is given by

$$
y_{k}=\tilde{l}_{k}+\left(M-\frac{T}{1+T}-\sum_{i=1}^{M} \tilde{l}_{i}\right)-\sum_{i=k+1}^{M}\left(w-\tilde{l}_{i}\right)
$$

Proof. In order to simplify the presentation, we rewrite the constraints in (8) as

$$
\forall i, q_{i} \leq y_{i} \leq w, \quad Q \triangleq \sum_{i=1}^{M} y_{i}=M-\frac{T}{1+T}
$$

where $q_{i} \triangleq \max \left\{\tilde{l}_{i}, 0\right\}$ and $w \triangleq 1-\varphi$. It is easy to see that $q_{i} \leq q_{i+1}$ and $Q \geq \sum_{i=1}^{M} q_{i}$, due to their definitions. To obtain the optimal vector $\mathbf{y}^{*}$, we employ an induction argument (similarly as in [6]). 
1. If $Q=\sum_{i=1}^{M} q_{i}, \forall i, y_{i}^{*}=q_{i}$ must be satisfied.

2. Increasing $Q$ with a small value $\left(0<\Delta \leq w-q_{M}\right)$, i.e., $Q=\sum_{i=1}^{M} q_{i}+\Delta$, the vector $\mathbf{y}^{*}$ must be structured as

$$
\begin{aligned}
& y_{i}^{*}=q_{i}, \quad i=1,2, \cdots, M-1, \\
& y_{M}^{*}=q_{M}+\Delta .
\end{aligned}
$$

The proof of this result is trivial, and is omitted for brevity.

3. Further increase $Q\left(w-q_{M}<\Delta \leq \sum_{i=M-1}^{M}\left(w-q_{i}\right)\right)$, then the vector $\mathbf{y}^{*}$ will be

$$
\begin{aligned}
& y_{i}^{*}=q_{i}, \quad i=1,2, \cdots, M-2, \\
& y_{M-1}^{*}=q_{M-1}+\Delta-\left(w-q_{M}\right), \\
& y_{M}^{*}=w .
\end{aligned}
$$

4. If $\sum_{i=M-1}^{M}\left(w-q_{i}\right)<\Delta \leq \sum_{i=M-2}^{M}\left(w-q_{i}\right)$, we have

$$
\begin{aligned}
& y_{i}^{*}=q_{i}, \quad i=1,2, \cdots, M-3, \\
& y_{M-2}^{*}=q_{M-2}+\Delta-\sum_{i=M-1}^{M}\left(w-q_{i}\right), \\
& y_{M-1}^{*}=y_{M}^{*}=w .
\end{aligned}
$$

5. Generally, if $\sum_{i=k+1}^{M}\left(w-q_{i}\right)<\Delta \leq \sum_{i=k}^{M}\left(w-q_{i}\right)$, the most majority $\mathbf{y}^{*}$ is

$$
\begin{aligned}
& y_{i}^{*}=q_{i}, \quad i=1,2, \cdots, k-1, \\
& y_{k}^{*}=q_{k}+\Delta-\sum_{i=k+1}^{M}\left(w-q_{i}\right), \\
& y_{k+1}^{*}=\cdots=y_{M}^{*}=w .
\end{aligned}
$$

Now, it is clear that the optimal $\mathbf{y}^{*}$ is related to the value of $Q$, and is structured as

$$
\mathbf{y}^{*}=[\underbrace{q_{1}, \cdots, q_{k-1}}_{k-1}, y_{k}, \underbrace{w, \cdots, w}_{M-k}]^{T},
$$

where the value of $k$ is determined by the following inequality

$$
\sum_{i=k+1}^{M}\left(w-q_{i}\right)<M-\frac{T}{1+T}-\sum_{i=1}^{M} q_{i} \leq \sum_{i=k}^{M}\left(w-q_{i}\right)
$$

and $y_{k}$ is given by

$$
y_{k}=q_{k}+\left(M-\frac{T}{1+T}-\sum_{i=1}^{M} q_{i}\right)-\sum_{i=k+1}^{M}\left(w-q_{i}\right) .
$$

Recall that $q_{i}=\max \left\{\tilde{l}_{i}, 0\right\}$ and $\tilde{l}_{i} \leq \tilde{l}_{i+1}$. There always exists a value of $n(0 \leq n \leq k-1)$ such that

$$
\left\{\begin{array}{l}
q_{i}=0, i \leq n \\
q_{i}=\tilde{l}_{i}, i>n
\end{array}\right.
$$

Consequently, the optimal solution may be rewritten as

$$
\mathbf{y}^{*}=[\underbrace{0, \cdots, 0}_{n}, \underbrace{\tilde{l}_{n+1}, \cdots, \tilde{l}_{k-1}}_{k-n-1}, y_{k}, \underbrace{w, \cdots, w}_{M-k}]^{T} .
$$

On the other hand, it should be noted that $y_{i}=\frac{1+T-x_{i}}{1+T}>$ $\frac{1}{1+T}>0$, which implies $n$ must be 0 . Thus, we achieve desired results in Theorem 1. Additionally, we know that for the case of $\tilde{l}_{i}<0$ or, equivalently, $l_{i}>T+1$, if there exists any feasible solution it must be $[w, w, \cdots, w]^{T}$.

Drawn from above discussions, the following theorem can be obtained.

Theorem 2. If there is any solution to problem (7), it is structured as

$$
\mathbf{x}=[\underbrace{l_{1}, \cdots, l_{k-1}}_{k-1}, x_{k}, \underbrace{\varphi(1+T), \cdots, \varphi(1+T)}_{M-k}]^{T}
$$

where $T=\sum_{i=1}^{M}[\mathbf{x}]_{i}, k$ is determined by (11), and $x_{k}$ equals

$$
\begin{aligned}
x_{k} & =(1+T)\left(1-y_{k}\right) \\
& =(1+T)(1-(M-k) \varphi)-\sum_{i=1}^{k-1} l_{i}-1 .
\end{aligned}
$$

\subsection{Our improved method}

Although the solution structure (18) has been addressed in [5], the relationship among $k, x_{k}$, and $T$ is not well revealed due to the limitation of the proof procedure therein. According to the results in [5], a triplet $\left(k, x_{k}, T\right)$ can be determined by any two of its elements, for example $k$ and $x_{k}$. Our new derivation shows that $\left(k, x_{k}, T\right)$ in fact can be simply determined by a single element $T$, while $k$ and $x_{k}$ can be obtained by (11) and (19) given $T$. Therefore, the candidate solution of (18) can be evaluated over different values of $T$ instead of $\left(k, x_{k}\right)$. As will be shown later, this plays an important role in reducing the computational complexity of our improved method.

A numerical search for different values of $k$ is used in [3] to find the optimal $x_{k}$, and the search is reduced to a finite number of one-dimensional spaces. The search is further reduced in [5] from a set of one-dimensional spaces to a finite set of points by employing the following lemma.

Lemma 2. In the optimal solution to problem (7), $x_{k}$ must accept one of the marginal values given in 


$$
\left\{\begin{aligned}
x_{k} & \leq \min \left\{l_{k},\left(X_{\max }+1\right)[1-(M-k) \varphi]-L-1, l_{M} \frac{1-(M-k) \varphi}{\varphi}-L-1\right\} \\
x_{k} & \geq \frac{\varphi(L+1)}{1-(M-k+1) \varphi}
\end{aligned}\right.
$$

where $L \triangleq \sum_{i=1}^{k-1} l_{i}$ (see Theorem 3 and Theorem 4 in [5]).

Applying Lemma 2, it is clear that the process of finding all the possible power allocations requires checking $k$, $1 \leq k \leq M$, while trying two marginal values given in (20). Therefore, almost $2 M$ candidate points need to be searched in the algorithm proposed in [5]. However, we note that if the constraints in Lemma 2 are rearranged, a more efficient method can be derived. The key observations for the development of our improved method are:

1. $x_{k}$ is a monotonic increasing function of $T$ and vice versa (see (19)).

2. If the optimal $x_{k}$ accepts one of the marginal values given in (20), $x_{k}$ must be one of the values among $\left\{l_{k},\left(X_{\max }+1\right)[1-(M-k) \varphi]-L-1, l_{M} \frac{1-(M-k) \varphi}{\varphi}\right.$ $\left.-L-1, \frac{\varphi(L+1)}{1-(M-k+1) \varphi}\right\}$.

3. Given $T$, the values of $k$ and $x_{k}$ are uniquely determined and vice versa (the relationship among $k$, $x_{k}$, and $T$ is given in Theorem 2). Therefore, searching candidate values of $T$ instead of $k$ and $x_{k}$ does not result in any loss of optimality.

Keeping these observations in mind, we now proceed to derive our efficient method based on the following theorem.

Theorem 3. In the optimal solution to problem (7), $T$ must accept one of the candidate values given by

$$
\begin{aligned}
& \left\{T_{k} \mid T_{k}<\left\{X_{\max }, \frac{l_{M}}{\varphi}-1\right\}, k=0,1, \cdots, M\right\} \bigcup \\
& \min \left\{X_{\max }, \frac{l_{M}}{\varphi}-1\right\}
\end{aligned}
$$

where $T_{k} \triangleq \frac{\sum_{i=1}^{k} l_{i}+(M-k) \varphi}{1-(M-k) \varphi}$ and $T_{k}<T_{k+1}$.

Proof. Relying on Observation 2 and 3, it is clear that proving the above theorem is equivalent to specifying the values of $T$ corresponding to the candidate values of $k$ and $x_{k}$, where $x_{k} \in\left\{l_{k},\left(X_{\max }+1\right)[1-(M-k)\right.$ $\left.\varphi-L-1], l_{M} \frac{1-(M-k) \varphi}{\varphi}-L-1, \frac{\varphi(L+1)}{1-(M-k+1) \varphi}\right\}$ and $1 \leq$ $k \leq M$. To gain some insight, we now discuss the possible values of $x_{k}$, respectively.
1. For the case of $x_{k}=l_{k}$, based on (19), the corresponding $T$ is given by

$$
\begin{aligned}
T & =\frac{L+x_{k}+(M-k) \varphi}{1-(M-k) \varphi} \\
& =\frac{\sum_{i=1}^{k} l_{i}+(M-k) \varphi}{1-(M-k) \varphi} \triangleq T_{k}
\end{aligned}
$$

where $1 \leq k \leq M$. Thus, the candidate values of $T$ in this case are given by

$$
\mathbb{T}_{1}=\left\{T_{k} \mid k=1, \cdots, M\right\}
$$

2. For the case of

$x_{k}=\left(X_{\max }+1\right)[1-(M-k) \varphi]-L-1$, using the expression for $x_{k}$ in (19), we obtain

$$
\begin{aligned}
& \left(X_{\max }+1\right)[1-(M-k) \varphi]-L-1=(1+T) \\
& (1-(M-k) \varphi)-L-1
\end{aligned}
$$

or, equivalently, $T=X_{\max }$. Hence, in this case, the candidate set of $T$ is

$$
\mathbb{T}_{2}=\left\{X_{\max }\right\}
$$

3. For the case of $x_{k}=l_{M} \frac{1-(M-k) \varphi}{\varphi}-L-1$, similarly, we get

$$
l_{M}=\varphi(1+T)
$$

Consequently, the candidate set is given as

$$
\mathbb{T}_{3}=\left\{\frac{l_{M}}{\varphi}-1\right\}
$$

4. For the case of $x_{k}=\frac{\varphi(L+1)}{1-(M-k+1) \varphi}$, we have

$$
\begin{aligned}
T & =\frac{L+x_{k}+(M-k) \varphi}{1-(M-k) \varphi} \\
& =\frac{\sum_{i=1}^{k-1} l_{i}+(M-(k-1)) \varphi}{1-(M-k+1) \varphi}
\end{aligned}
$$

where $1 \leq k \leq M$. Therefore, the candidate set of $T$ is

$$
\mathbb{T}_{4}=\left\{T_{k} \mid k=0, \cdots, M-1\right\}
$$


Putting all the possible $T$ together and using the fact that any feasible $T$ satisfies $T<X_{\max }$ and $T<\frac{l_{M}}{\varphi}-1$, we are able to derive the whole candidate set of $T$ as

$$
\begin{aligned}
& \mathbb{T}=\left.\left\{\mathbb{T}_{1} \bigcup \mathbb{T}_{2} \bigcup \mathbb{T}_{3} \bigcup \mathbb{T}_{4}\right\}\right|_{T \leq \min }\left\{X_{\max }, \frac{l_{M}}{\varphi}-1\right\} \\
&=\left\{T_{k} \mid T_{k}<\min \left\{X_{\max }, \frac{l_{M}}{\varphi}-1\right\}, k=0,1, \cdots, M\right\} \\
& \bigcup \min \left\{X_{\max }, \frac{l_{M}}{\varphi}-1\right\} .
\end{aligned}
$$

For any $T_{k}$ and $T_{k+1}$, the solutions are structured as

$$
\begin{aligned}
\mathbf{x}_{k}= & {[\underbrace{l_{1}, \cdots, l_{k-1}}_{k-1}, l_{k}, \underbrace{\varphi\left(1+T_{k}\right), \cdots, \varphi\left(1+T_{k}\right)}_{M-k}]^{T}, } \\
\mathbf{x}_{k+1}= & {[\underbrace{l_{1}, \cdots, l_{k}}_{k}, l_{k+1}, \underbrace{\varphi\left(1+T_{k+1}\right), \cdots, \varphi\left(1+T_{k+1}\right)}_{M-k-1}]^{T} }
\end{aligned}
$$

Further, we note that $\mathbf{x}_{k}$ can be written as

$$
\mathbf{x}_{k}=[\underbrace{l_{1}, \cdots, l_{k}}_{k}, \varphi\left(1+T_{k}\right), \underbrace{\varphi\left(1+T_{k}\right), \cdots, \varphi\left(1+T_{k}\right)}_{M-k-1}]^{T}
$$

In this form, it is easy to see that the structures of (32) and (33) are equivalent and $\varphi\left(1+T_{k}\right) \leq l_{k+1}$ (which follows from the first constraint of (7)). So, according to Observation 1 and

$$
\varphi\left(1+T_{k}\right)=\left[\mathbf{x}_{k}\right]_{k+1}<\left[\mathbf{x}_{k+1}\right]_{k+1}=l_{k+1},
$$

we achieve the desired result that $T_{k}<T_{k+1}$. It should be noted that since $T_{k}<T_{k+1}$, there always exists a unique $j$ such that

$$
T_{j} \leq \min \left\{X_{\max }, \frac{l_{M}}{\varphi}-1\right\} \leq T_{j+1}
$$

which means the structure of solution for the case of $T=$ $\min \left\{X_{\max } \frac{l_{M}}{\varphi}-1\right\}$ has been determined by the value of $j$.

Obviously, the candidate set of $T$ contains less than $M+2$ points that need to be searched to find the optimal solution. Based on these conclusions, our improved algorithm is outlined as follows. Compared with the original $2 M$ search points, less than half of the computational calculations are required by our method in the worst case $^{\mathrm{a}}$.

1. Initialization: $X_{\max }=P_{R}^{\max } / I, \varphi=\frac{\gamma}{1+\gamma}$, $l_{i}=p_{\max } g_{i} / I$.

2. For all $0 \leq k \leq M$, do the following: (a) Compute $T_{k}$ as

$$
T_{k}=\frac{\Sigma_{i=1}^{k} l_{i}+(M-k) \varphi}{1-(M-k) \varphi} .
$$

(b) If $T_{k} \leq \min \left\{X_{\max }, \frac{l_{M}}{\varphi}-1\right\}$, compute $\phi_{k}$ as

$$
\phi_{k}=\frac{(1-\varphi)^{(M-k)}}{\left(1+T_{k}\right)^{k}} \prod_{i=1}^{k}\left(1+T_{k}-l_{i}\right) \text {. }
$$

(c) If $T_{k}>\min \left\{X_{\max }, \frac{l_{M}}{\varphi}-1\right\}$, compute $\phi_{k}$ as

$$
\begin{aligned}
\phi_{k}= & \frac{\left(\left(1+\min \left\{X_{\max }, \frac{l_{M}}{\varphi}-1\right\}\right)(M-k) \varphi+\sum_{i=1}^{k-1} l_{i}+1\right)(1-\varphi)^{(M-k)}}{\left(1+\min \left\{X_{\max }, \frac{l_{M}}{\varphi}-1\right\}\right)^{k}} \\
& \cdot \prod_{i=1}^{k-1}\left(1+\min \left\{X_{\max }, \frac{l_{M}}{\varphi}-1\right\}-l_{i}\right)
\end{aligned}
$$

and go to step 3 , else continue (go to step 2a).

3. Find the smallest $\phi_{k}$, denoted as $\phi^{*}$, and retrieve the corresponding $\mathbf{x}$.

4. Output the $p_{i}$ and $C$ :

$$
\begin{aligned}
p_{i} & =I x_{i} / g_{i}, \\
C & =-\log _{2} \phi^{*} .
\end{aligned}
$$

\section{Discussions:}

- If the value of $\min \left\{X_{\max }, \frac{l_{M}}{\varphi}-1\right\}$ is small, according to our method, very few points need to be searched. In particular, if $T_{0} \leq \min \left\{X_{\max }, \frac{l_{M}}{\varphi}-1\right\} \leq T_{1}$, just a point $T=\min \left\{X_{\max }, \frac{l_{M}}{\varphi}-1\right\}$ needs to be searched. However, if $\min \left\{X_{\max }, \frac{l_{M}}{\varphi}-1\right\}<T_{0}$, there is no feasible solution.

- If the value of $\min \left\{X_{\max }, \frac{l_{M}}{\varphi}-1\right\}$ is large, e.g., $\min \left\{X_{\max }, \frac{l_{M}}{\varphi}-1\right\} \geq T_{M}$, it is clear that the third constraint in (6) does not affect the final solution and can be removed from the optimization problem.

\section{Simulation result}

The simulation results in this section illustrate the performance of our method. The computer codes are developed in MATLAB 7.8.0 and are run on a PC with an Intel Core2 $2.66 \mathrm{GHz}$ CPU and 4GB of RAM. We will compare the proposed method to the original methods in [5] (the original method 1 considers the same optimization problem as our method, while the original method 2 considers a new optimization problem which includes an additional capacity upper bound for each MS) and the max-min method in [6].

To justify whether the sum capacity achieved by the proposed method is consistent with the original method 1 , in simulation 1 , we first consider the same scenario used in 
Table 1 Comparison of optimal solutions between our method and the original method 1

\begin{tabular}{lccccc}
\hline Station & $\boldsymbol{g}_{\boldsymbol{i}}\left(\mathbf{\times} \mathbf{1 0}^{-\mathbf{1 2}}\right)$ & $\boldsymbol{p}_{\boldsymbol{i}}$ (original) & $\boldsymbol{p}_{\boldsymbol{i}}$ (improved) & $\boldsymbol{C}_{\boldsymbol{i}}$ (original) & $\boldsymbol{C}_{\boldsymbol{i}}$ (improved) \\
\hline 1 & 0.52 & 46.6616 & 46.6616 & 2.3606 & 0.3606 \\
2 & 0.018 & 5.2767 & 5.2767 & 0.0046 & 0.0046 \\
3 & 0.016 & 5.9363 & 5.9363 & 0.0046 & 0.0046 \\
4 & 0.0091 & 10.4375 & 10.4375 & 0.0046 & 0.0046 \\
5 & 0.0082 & 11.5831 & 11.5831 & 0.0046 & 0.0046 \\
6 & 0.0081 & 11.7261 & 11.7261 & 0.0046 & 0.0046 \\
7 & 0.0075 & 12.6642 & 12.6642 & 0.0046 & 0.0046 \\
8 & 0.0059 & 16.0985 & 16.0985 & 0.0046 & 0.0046 \\
9 & 0.0059 & 16.0985 & 16.0985 & 0.0046 & 0.0046 \\
10 & 0.0045 & 21.1070 & 21.1070 & 0.0046 & 0.0046 \\
\hline
\end{tabular}

[5], where $\gamma=-50 \mathrm{~dB}, I=-113 \mathrm{dBm}, P_{R}^{\max }=-106 \mathrm{dBm}$ and $p_{\max }=23 \mathrm{dBm}\left(\eta \mathrm{dBm} \triangleq 10^{\frac{\eta}{10}} \times 10^{-3}\right) . M$ data MSs are randomly dropped with a uniform distribution over a hexagonal cell. Assume that there is a sample where $M=10$, and the values of $g_{i}$ are $10^{-12} \times$ [0.52, 0.018, 0.016, 0.0091, 0.0082, 0.0081, 0.0075, 0.0059, $0.0059,0.0045]$ [5]. Table 1 shows the optimal solutions for our method and the original method 1, including the power allocation $p_{i}$ and capacity $C_{i}, i=1,2, \cdots, M$. The results illustrate that two methods achieve the same results.

To testify the performance of an algorithm, we should prove that the algorithm can work for a wide variety of scenarios, which mainly depend on the number of MSs, their locations, and the services they want to use. In simulation 2, we run some Monte Carlo simulations to show the sum capacity achieved by our proposed method. We work on a practical scenario from 3GPP2 [11], where the configurations used in this simulation are summarized in Table 2. $M$ MSs are randomly dropped with a uniform distribution over a hexagonal cell, and the base station is located at the center of the cell. A set of $M$ dropped users is said to be valid if the minimum QoS requirements are achievable. When the set is invalid, the entire users are redropped. Each path gain, $g_{i}$, takes into account distance loss and log-normal shadowing. However, fast fading and power control aspects are not considered in the simulation because the optimization problem does not consider the time-varying condition. The rise-over-thermal (ROT) limiting the total received power at the base station is defined as $\left(P_{R}^{\max }+\sigma_{\text {th }}^{2}\right) / \sigma_{\text {th }}^{2}$, where $\sigma_{\text {th }}^{2}$ is the thermal noise variance at the base station. Figures 1 and 2 show the sum capacity achieved by transmission strategies versus the number of MSs with ROT being 7 and $10 \mathrm{~dB}$, respectively. All the results are obtained by averaging the sum capacity over 10,000 channel realizations, and the optimal power allocations are optimized for each channel realization. As can be seen from the figures, the sum capacity curves for our method and the original method 1 coincide, which again illustrates that our method always achieves the same solution as the original method 1 . These two methods always outperform the original method 2, as well as the max-min method, in terms of the sum capacity. The reasons are (1) the feasible region of the new optimization problem, which includes an additional capacity upper bound for each MS $\left(C_{i}<0.3\right)$, is less than the feasible region of (6) and (2) the max-min method is designed to maximize the minimum rate rather than the sum capacity. Moreover, we observe some similar results about inconsistent reduction of the system capacity as in $[3,4]$. Without a QoS requirement, the sum capacity increases as $M$ increases because of the multi-user diversity. In contrary, if there is a QoS requirement (e.g., -21 $\mathrm{dB}$ or $-18 \mathrm{~dB}$ ), the sum capacity decreases by increasing $M$. The reason is that the statistics of the poor channel gets worse as $M$ increases. Hence, meeting the QoS of the poor channel users will drag the system capacity. Note that QoS requirements of $-21 \mathrm{~dB}$ and $-18 \mathrm{~dB}$ are related to the typical SINR level for $9.6 \mathrm{Kbps}$ traffic channel throughput maintenance and reverse pilot channel maintenance, respectively $[4,12]$.

Table 2 Configurations for simulation 2

\begin{tabular}{lc}
\hline Configurations & Value \\
\hline Cell structure & Single hexagonal cell \\
Cell Radius & $1.0 \mathrm{~km}$ \\
Antenna radiation & Omni-directional \\
Distance loss (propagation) model & $28.6+35 \log _{10}(d) \mathrm{dB}$ \\
Log-normal shadowing standard deviation & $8.9 \mathrm{~dB}$ \\
Maximum transmit power $\left(p_{\max }\right)$ & $0.2 \mathrm{Watt}$ \\
Implementation loss & $0 \mathrm{~dB}$ \\
Thermal noise variance & $1.547 \times 10^{-14}$ \\
& Watt $(=-138.1 \mathrm{~dB})$ \\
\hline
\end{tabular}




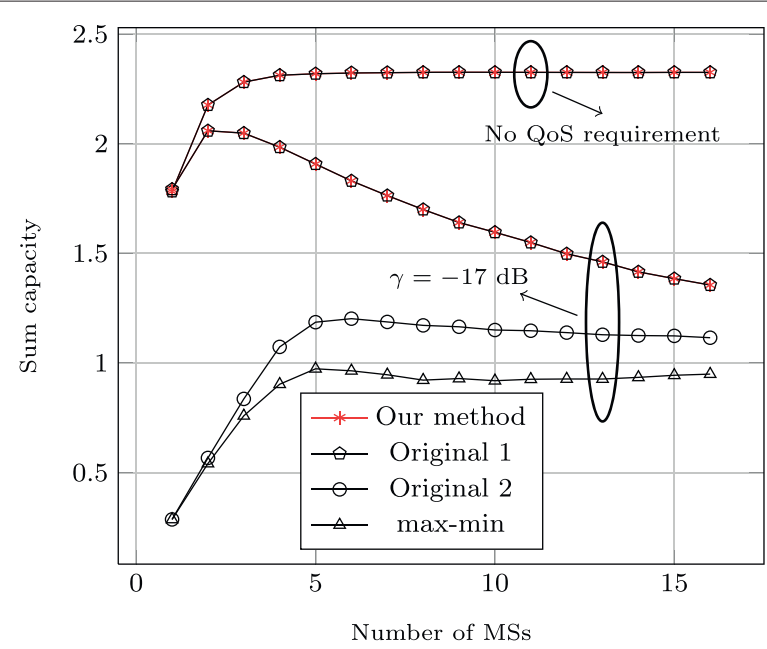

Figure 1 Sum capacity for different QoS constraints with ROT being $7 \mathrm{~dB}$.

Simulation 3 illustrates capacity shares for the major user, where the same scenario is considered as in simulation 2 . Figures 3 and 4 show the capacity share of the major user achieved by transmission strategies versus the QoS requirement $\gamma$ with ROT being 7 and $10 \mathrm{~dB}$, respectively. All the results are obtained by averaging $\max _{i} C_{i} / C$ over 10,000 channel realizations, and the optimal power allocations are optimized for each channel realization. From the figures, we observe that: (1) the optimization problem (6) produces an unfair solution that is dominantly dependent on the major user, if the QoS requirement $\gamma$ is low, (2) the new optimization problem that includes an additional capacity upper bound for each MS $\left(C_{i}<0.3\right)$ will improve the fairness, (3) the max-min method achieves the most

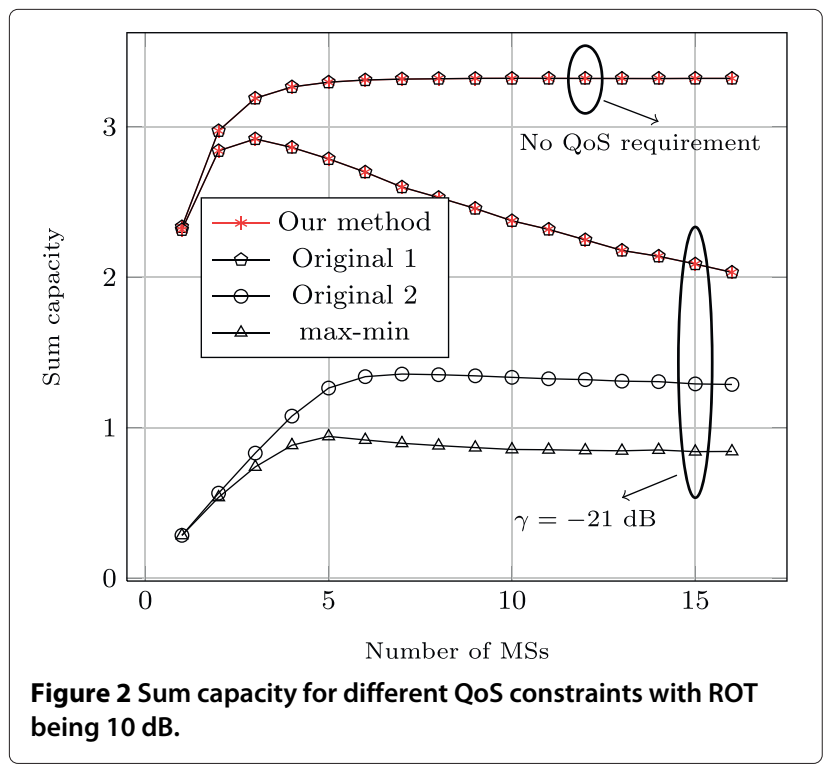

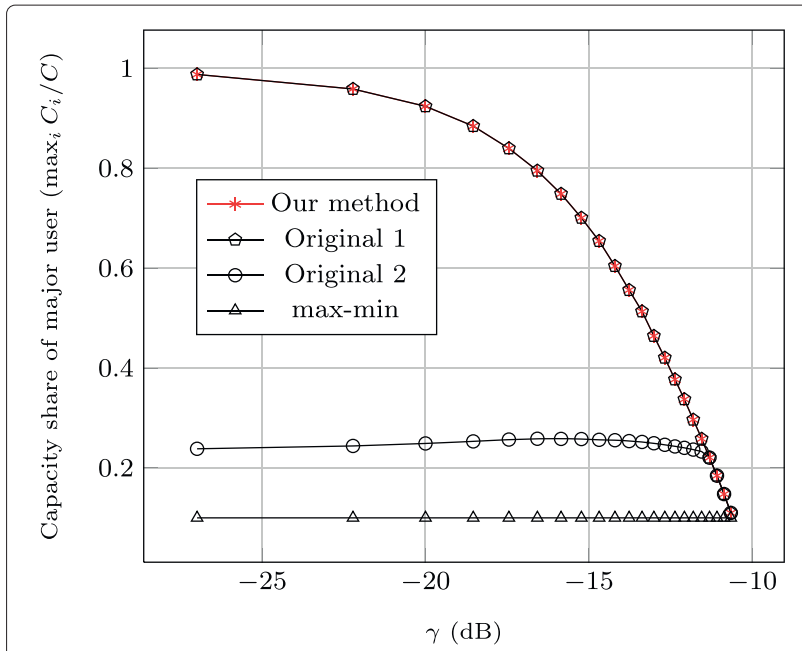

Figure 3 Capacity share of the major user with ROT being $7 \mathrm{~dB}$.

fair solution because it is designed for this purpose, and (4) the gap of capacity shares among our method, the original method 2 and the max-min method trend to close in higher QoS requirement regime.

In simulation 4, we run another Monte Carlo simulation to evaluate the exact computational cost of our proposed method. Consider the same scenario in simulation 2, except that the QoS requirement is set to $-21 \mathrm{~dB}$ and the number of MSs changes from 10 to 200. Figure 5 gives the running time of our method and the method in [5] versus the number of MSs with ROT being $10 \mathrm{~dB}$. All the results are obtained by summing the running time over 10,000 channel realizations, and the optimal power allocations are optimized for each channel realization. The simulation results demonstrate that our proposed

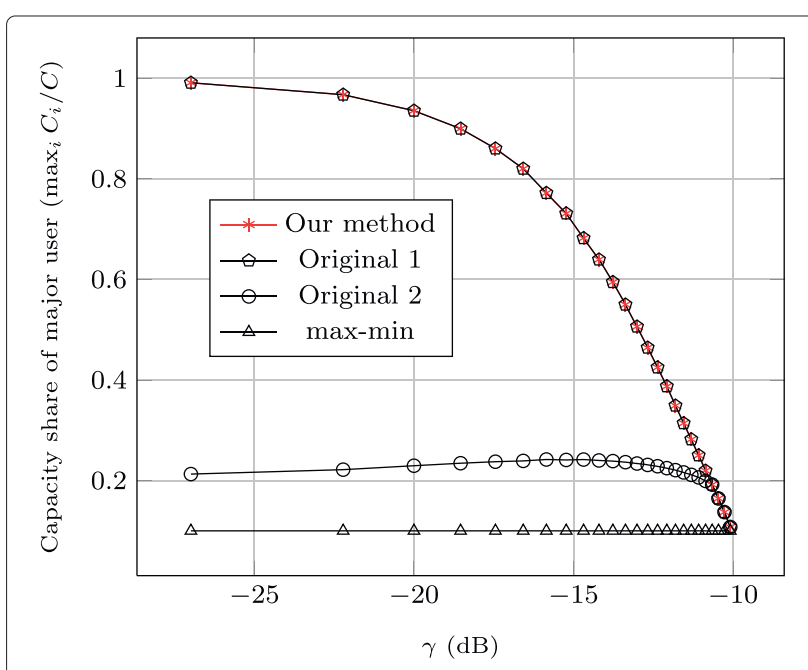

Figure 4 Capacity share of the major user with ROT being $10 \mathrm{~dB}$. 


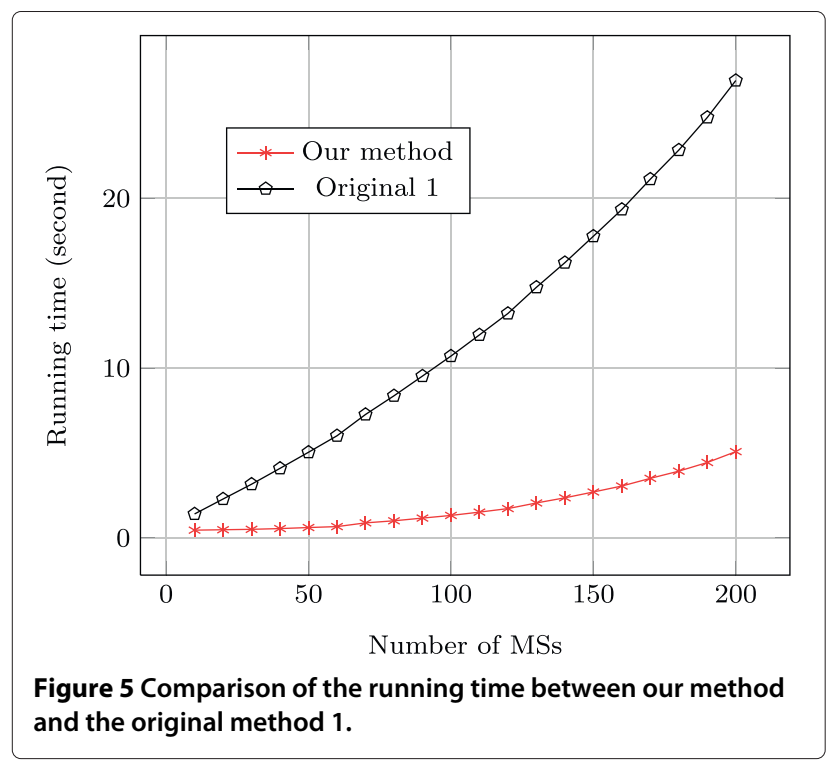

method has a much lower computational complexity than the state-of-art method under comparison.

\section{Conclusions}

Optimal power allocation for maximizing the MAC capacity of non-real-time users with QoS constraints has been investigated in this paper. Based on majorization theory, we show that the optimal structure of the solution can be easily obtained. Additionally, by rearranging the constraints in Lemma 2 and restricting the attention to searching candidate values of $T$ instead of $k$ and $x_{k}$, a more efficient method is derived. Compared with the original method, less than half of the computational cost is required by our method in the worst case. Simulation results demonstrate the effectiveness of our method. For future work, it will be interesting to see if one can extend the method to the case of joint power constraints [13,14].

\section{Endnote}

${ }^{a}$ The computational cost of the existing method is of $\mathcal{O}\left(M^{2}\right)$ with $8 M^{2}$ flops in the worst case [5]. The computational cost of our method is of the same order but with $1.5 M^{2}$ flops in the worst case. Step $2 \mathrm{a}$ and step $2 \mathrm{~b}$ totally require $0.5 M^{2}$ and $M^{2}$ flops, respectively. As Step $2 \mathrm{c}$ is executed once, its computational cost is neglectable.

\section{Competing interests}

The authors declare that they have no competing interests.

\section{Acknowledgements}

This work was supported in part by the National Natural Science Foundation of China (61102054), the Open Research Fund of National Mobile

Communications Research Laboratory, Southeast University (No. 2013D08), the Jiangsu Planned Projects for Postdoctoral Research Funds (No. 1302004A), the Project funded by China Postdoctoral Science Foundation, and the Project Foundation for Priority Academic Program Development (PAPD) of Jiangsu Higher Education Institutions.

\section{Author details}

${ }^{1}$ School of Electrical and Information Engineering, Jiangsu University, Zhenjiang 212013, China. ${ }^{2}$ National Mobile Communications Research Laboratory, Southeast University, Nanjing 210096, China. ${ }^{3}$ Nettech Technology (Suzhou) Co., Ltd., Suzhou 215131, China. ${ }^{4}$ School of Electronic and Information Engineering, Soochow University, Suzhou 215006, China.

Received: 27 December 2013 Accepted: 4 July 2014

Published: 15 July 2014

\section{References}

1. A Muqattash, M Krunz, S Tao, Performance enhancement of adaptive orthogonal modulation in wireless CDMA systems. IEEE J. Selec. Areas Commun. 24(3), 565-578 (2006)

2. SA Jafar, A Goldsmith, Adaptive multirate CDMA for uplink throughput maximization. IEEE Trans. Wireless Commun. 2(2), 218-228 (2003)

3. SJ Oh, ACK Soong, QoS-constrained information-theoretic sum capacity of reverse link CDMA systems. IEEE Trans. Wireless Commun. 5(1), 3-7 (2006)

4. D Zhang, SJ Oh, N Bhushan, Optimal resource allocation for data service in CDMA reverse link. IEEE Trans. Wireless Commun. 6(10), 3648-3656 (2007)

5. A Abadpour, AS Alfa, ACK Soong, Closed form solution for maximizing the sum capacity of reverse-link CDMA system with rate constraints. IEEE Trans. Wireless Commun. 7(4), 1179-1183 (2008)

6. J Dai, Z Ye, XXu, Power allocation for maximizing the minimum rate with QoS constraints. IEEE Trans. Vehic. Technol. 58(9), 4989-4996 (2009)

7. DP Palomar, JM Cioffi, MA Lagunas, Joint Tx-Rx beamforming design for multicarrier MIMO channels: a unified framework for convex optimization. IEEE Trans. Signal Process. 51(9), 2381-2401 (2003)

8. AW Marshall, I Olkin, Inequalities: Theory of Majorization and Its Applications. (Academic, New York, 1979)

9. DP Palomar, Y Jiang, MIMO Transceiver Design via Majorization theory. Foundations and trends in communications and information theory. 3(4-5), 331-551 (2006)

10. SJ Oh, Resource allocation in wireless networks. Ph. D. dissertation, Univ of Michigan (2000)

11. Working Group 5 Evaluation Ad-Hoc Group, 1xEV-DV evaluation methodology - Addendum (V6). Third Generation Partnership Project 2 (3GPP2), Contribution C50-20010820-026 (2001)

12. S Chakravarty, R Pankaj, E Esteves, An algorithm for reverse traffic channel rate control for CDMA2000 high rate packet data systems, in Proc. IEEE Globecom, (2001), pp. 3733-3737

13. J Dai, C Chang, X Xu, Z Ye, Linear precoder optimization for MIMO systems with joint power constraints. IEEE Trans. Commun. 60(8), 2240-2254 (2012)

14. J Dai, X Bao, Homotopy algorithm for energy-efficient MIMO systems with joint power constraints. IEEE Wireless Commun. Lett. 3(2), 121-124 (2014)

doi:10.1186/1687-6180-2014-113

Cite this article as: Dai and Chang: Power allocation for maximizing the MAC capacity via majorization. EURASIP Journal on Advances in Signal Processing 2014 2014:113.

\section{Submit your manuscript to a SpringerOpen ${ }^{\circ}$ journal and benefit from:}

- Convenient online submission

- Rigorous peer review

- Immediate publication on acceptance

- Open access: articles freely available online

- High visibility within the field

- Retaining the copyright to your article

Submit your next manuscript at $>$ springeropen.com 